\title{
The role of pleiotropy vs signaller-receiver gene epistasis in life history trade-offs: dissecting the genomic architecture of organismal design in social systems
}

\author{
B Sinervo ${ }^{1,2,3}$, J Clobert ${ }^{2,3}$, DB Miles ${ }^{4}$, A McAdam ${ }^{5,6}$ and LT Lancaster ${ }^{1}$ \\ ${ }^{1}$ Department of Ecology and Evolutionary Biology, University of California, Santa Cruz, CA, USA; ${ }^{2}$ Laboratoire Evolution et Diversité \\ Biologique, Bâtiment 4R3, UMR 5174 CNRS, Université Paul Sabatier, Toulouse Cedex, France; ${ }^{3}$ Station d'Ecologie Expérimentale du \\ CNRS à Moulis, Saint-Girons, France; ${ }^{4}$ Department of Biology, Ohio University, Athens, OH, USA; ${ }^{5}$ Department of Fisheries and

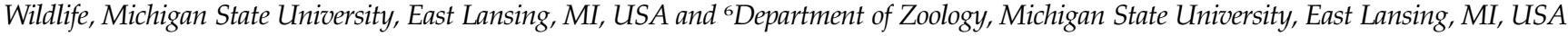

\begin{abstract}
Traditional life history theory ignores trade-offs due to social interactions, yet social systems expand the set of possible trade-offs affecting a species evolution-by introducing asymmetric interactions between the sexes, age classes and invasion of alternative strategies. We outline principles for understanding gene epistasis due to signaller-receiver dynamics, gene interactions between individuals, and impacts on life history trade-offs. Signaller-receiver epistases create trade-offs among multiple correlated traits that affect fitness, and generate multiple fitness optima conditional on frequency of alternative strategies. In such cases, fitness epistasis generated by selection can maintain linkage disequilibrium, even among physically unlinked loci. In
\end{abstract}

reviewing genetic methods for studying life history tradeoffs, we conclude that current artificial selection or gene manipulation experiments focus on pleiotropy. Multi-trait selection experiments, multi-gene engineering methods or multiple endocrine manipulations can test for epistasis and circumvent these limitations. In nature, gene mapping in field pedigrees is required to study social gene epistases and associated trade-offs. Moreover, analyses of correlational selection and frequency-dependent selection are necessary to study epistatic social system trade-offs, which can be achieved with group-structured versions of Price's (1970) equation. Heredity (2008) 101, 197-211; doi:10.1038/hdy.2008.64; published online 6 August 2008

Keywords: life history trade-offs; social system; Price equation; epistasis; pleiotropy; selection

\section{Introduction}

In the standard paradigm, life history theory assumes that genetic trade-offs arise from pleiotropy (Reznick et al., 2000), which is the effect of one gene on two or more traits. However, awareness of more complex genomic architectures is growing (Roff and Fairbairn, 2006). We discuss the underappreciated role that social interactions play in expanding the types and forms of trade-offs that may influence trait expression and fitness (Wolf et al., 1999). In social systems, interactions among genotypes of individuals generate fitness epistasis. The effect of genes on fitness of one individual is affected by those expressed by other individuals. In this view, the direct effect of genes on traits within individuals may be altered by the indirect effect of genes in neighbours (for example, 'neighbour-modulated fitness', Grafen, 2006). Therefore, the direct pleiotropic effect of a single gene can be altered by indirect gene interactions between

Correspondence: Professor B Sinervo, Department of Ecology and Evolutionary Biology, Earth and Marine Sciences Building, University of California, Santa Cruz, CA 95064, USA.

E-mail: sinervo@biology.ucsc.edu

Received 12 October 2007; revised 28 April 2008; accepted 15 May 2008; published online 6 August 2008 social individuals. Furthermore, social individuals interact through signaller loci and receiver loci, which are often caused by at least two or more different loci. Therefore, the indirect genetic effects of genes between individuals will ramify into complex gene interactions involving both pleiotropic and epistatic causes. This view is integrated in the literature on co-evolutionary interactions among species (Thompson, 2005), but not yet fully integrated into the study of life history theory.

In the field of behavioural ecology the role of signallerreceiver interactions are well understood, but few studies in behavioural ecology incorporate the full set of life history trade-offs that might arise, such as in the case of life history trade-offs that structure sexual selection (Kokko, 2001). Moreover, behavioural ecology focuses on phenotypes and only rarely on genotypes, and we argue that understanding the role of genes in governing life history trade-offs and social system trade-offs is crucial to a comprehensive understanding of the suite of organismal traits. In particular, we argue that epistatic gene interactions generate trade-offs among multiple trait complexes not predicted by pleiotropic effects alone, which tends to focus on within individual effects of single genes on two or more traits. We review the generality of epistasis in moulding trade-offs of organismal function, including life history trade-offs. These new views will be useful for 
researchers in the fields of life history theory and behavioural ecology, as well as for evolutionary geneticists that are curious about the special and general effects of genes for aggregation behaviour on fitness and the life history. Genetically based aggregation behaviours can also generate emergent properties that self-assemble novel social systems, particularly in the context of alternative social strategies.

Social trade-offs arise through the inherent conflict and cooperation between signallers and receivers. For example, kin selection is a social trade-off that is due to the costs of kin competition vs benefits of kin cooperation (Hamilton and May, 1977). The interplay between social and life history trade-offs is obvious in kin helpers of avian social systems (that is, often progeny), who forgo reproduction to help kin (that is, often parents or other sibs; Komdeur and Hatchwell, 1999), but these kin helpers may survive to inherit a high quality parental (or sib) territory. Kin helping (by progeny or sibs) results in a delayed age of reproduction for the helper, but this delay confers reproductive benefits for related individuals (for example, parents or sisters). Given the role of gene sharing in fostering cooperation, a principal cause of social system trade-offs is symmetry vs asymmetry in gene relatedness. Recent advances in behavioural ecology have identified interesting social systems in which individuals need not be related to foster aggregations and invoke evolutionary cooperation (for example, greenbeard loci) and we review some of these studies to illustrate the properties of gene sharing of aggregation alleles, which transcend genealogical relationships and kin selection.

We also provide formalisms for measuring selection on the genomic architecture of trade-offs. We elaborate on the utility of Price's (1970) equation in the analysis of signaller-receiver gene and fitness epistasis that shapes life history trade-offs of social species. We argue that Price's Equation can be used to partition trade-offs due to pleiotropy vs trade-offs due to signaller-receiver epistasis in social systems. If combined with game theory (for example, studies of frequency-dependent selection), the Price equation (PE) provides a rich tapestry upon which we can analyse the roles of social interactions in shaping life history trade-offs, such as those invoked by densitydependent competition, or those invoked by social tradeoffs related to competition vs cooperation. The frequency dependence of the neighbour-modulated fitness effects of genes can readily be incorporated into the PE.

The role of pleiotropy in generating trade-offs is best characterized with genetic inference (Reznick et al., 2000). Understanding gene epistasis is impossible without genetic inference. We compare and contrast the utility of various genetic approaches in elucidating the pleiotropic vs epistatic sources of life history trade-offs. In particular, we argue that gene mapping methods will be required to understand the role(s) of epistasis in life history trade-offs. Such gene mapping methods should be applied to field pedigrees to understand how complex epistatic and multi-trait trade-offs arise in natural contexts.

\section{The genetic source(s) of life history trade-offs}

The standard view of life trade-offs is that they arise from a genetic trade-off (Reznick et al., 2000). A genetic correlation between traits, which both positively affect fitness, but are negatively related to each other, is termed a negative genetic correlation.

Negative genetic correlations and thus genetically based life history trade-offs can have two very different causes. Trade-offs and negative genetic correlations can arise from pleiotropy in which a single gene controls the expression of two or more traits, or from selection that creates gametic phase linkage disequilibrium (LD) of physically linked and unlinked loci, a non-random association of alleles at many loci (Lynch and Walsh, 1998). It is often assumed that because segregation and recombination will erode $L D$, genetic correlations due to this cause cannot be responsible for life history tradeoffs. Situations do occur in which LD will persist, such as the evolution of social systems that evolve under frequency-dependent selection (that is, the frequency of individuals with the same or a different social strategy can alter the fitness outcome).

Our fundamental thesis is that all genetic trade-offs expressed in social systems, involve gene epistasis, not merely pleiotropy. Social systems are comprised of interactions among all potential actors such as the juveniles, adult males and adult females of a sexual species. Other examples of social systems include interacting bacteria and viruses, protista, multicellular organisms and cells in multicellular organisms. Much social behaviour is generated by signal and receiver loci. Signal loci will more often than not be due to different loci that activate behaviours in receivers. Therefore, signaller and receiver genes, which are inherent to social contexts, will result in epistasis of the genes present in one individual and those present in other social actors. Social interactions can alter resource allocation to competing life history functions. Thus, pleiotropy is still involved in trade-offs because loci controlling signals, chiefly honest signals, are pleiotropically linked to life history trade-offs by physiology, immune or endocrine systems. We first review classic life history trade-offs and other trade-offs of behavioural ecology. We then link these trade-offs to the different levels of epistasis (for example, gene, physiological, fitness). Finally, we review the genetic methods for uncovering the pleiotropic vs epistatic causes of life history and social system tradeoffs, including the utility of the Price's (1970) equation in studying social system trade-offs per se.

\section{Pleiotropy vs gene epistasis as a source of life history} and functional trade-offs

Classic life history trade-offs: Life history theories are typically expressed as gene pleiotropy, such as genes that simultaneously affect the egg size and number trade-off. Other life history trade-offs include costs of reproduction, a trade-off between current and future reproductive effort (Sinervo, 1999; Reznick et al., 2000; Zera and Harshman, 2000) and senescence (Rose and Charlesworth, 1981a,b; Charmantier et al., 2006). Senescence is thought to arise from a selective premium placed on alleles for early maturity, which have pleiotropic effects that shorten life span. Trade-offs are well studied in many natural systems (Sinervo, 1999; Zera and Harshman, 2000; Reznick et al., 2000; Roff and Fairbairn, 2006). There is however, evidence that life history trade-off geometries are complex and often 
involve more than the two traits listed above (Ferrière and Clobert, 1992; Sinervo, 1999; Ernande et al., 2004; Miller and Sinervo, 2007). For example, densitydependent competition can alter the form of the egg size-number trade-off that governs progeny survival and recruitment and thereby alter the costs of reproduction trade-off that governs adult reproduction and survival (Sinervo and Svensson, 2002; Lancaster et al., 2008).

Social trade-offs, the life history and functional integration: Although classic life history trade-offs are often quite well resolved in a given organism, detecting life history trade-offs which also involve explicit behavioural and social traits has been hampered by a complicated interplay between Resource Holding Potential (RHP) that enhances fitness by aggressiveness (Maynard Smith, 1982), relative to alternative strategies that enhance fitness through crypsis (Shuster and Wade, 2003). Life history theory predicts that RHP traits will express pleiotropic costs on survival. In contrast sexual selection theory (Kokko et al., 2006) predicts that signals for male competition or mate attraction will be coupled epistatically to physiological systems like immune function (Ahtiainen et al., 2005) and performance (Miles et al., 2007) due to the energetic demands mate competition or mate attraction.

To understand the links between behavioural traits like signals and life history traits, it is key to understand the functional integration (Wagner and Altenberg, 1996) among traits that contribute to each physiological system which affects the allocation of resources towards behaviours or other life history components. For example, loci for stamina that enhance RHP should be well integrated with loci for metabolic rate, given that stamina is energetically demanding (Miles et al., 2007), but enhanced stamina due to increased basal metabolic rate may draw resources away from immune function. Functional integration is thought to arise from correlational selection in which alternative strategies are matched for alleles at loci of many interacting physiological systems (Miles et al., 2007). Allocation to immune function and life history traits is related to alternative strategies listed above (Svensson et al., 2002). Thus resource allocation per se and life history trade-offs can have a social cause. These social types are a permanent fixture of fitness landscapes and very general for all organisms (Sinervo and Calsbeek, 2006; Sinervo et al., 2007). The impacts of these social types on the genomic architecture of life history trade-offs must be addressed.

\section{A syllogism on communication, frequency-dependent and correlational selection}

How does the evolution of signaller and receiver systems shape pleiotropy and/or epistasis? Signaller and receiver co-evolution is the basis of communication. Communication is defined to be behaviour(s) (for example, trait or signal) of an individual that impacts behaviour of another (or self). At least some loci that govern signals must differ from loci that govern signal reception (that is, not pleiotropic in effect). Moreover, fitness outcomes of behaviours and traits that are elicited in receivers, due to communication, are likely due to loci other than just signaller and receiver loci. For example, the interaction between RHP traits (and loci) and immune function traits (and loci), discussed above will obviously involve many physiological systems and many interacting loci. Social interactions and social systems result in three combinations of correlational selection: (1) betweengenes, within-senders which couples traits for signals and other traits (and loci) that enhance or diminish signal activity, (2) between-genes, within-receivers, which couples the traits of receiver(s) and behavioural traits initiated at signal reception and (3) between-genes of senders and receivers which involve their different traits/loci and co-evolutionary outcomes (for example, evolving meaning of a signal).

Each combination of genetic interaction among signaller-receivers has the potential to generate trade-offs among multiple life history traits. Correlational selection operating on many traits should functionally integrate the components of social systems, subject to the constraints of resource allocation and life history tradeoffs. For example, RHP traits should be coupled with physiological capacity, but physiological capacity may impact survival. In communication, correlational selection therefore results in inter and intra-individual selection (Sinervo and Calsbeek, 2006), which have cascading effects on life history trade-offs. Only in the case of sexual selection (Kokko et al., 2006), a signallerreceiver communication between mates, has this coupling been explored in any detail. Sexual selection evolves under Fisherian runaway process that can build a genetic correlation between female preference and male signals and genes for quality. Life history trade-offs are typically measured only as pairs of traits. The full trade-offs for both sexes have been ignored (Kokko, 2001). The multi-variate approach of correlational selection is required to assess these more complex trade-offs geometries, and this approach also requires an analysis of the form of social interactions.

Correlational selection on signaller traits and receiver responses are frequency dependent (Sinervo and Calsbeek, 2006). This is because the success of a given signal depends on the frequency of that signal in a population. The success of a given response by a receiver also depends on the frequency with which that response is used in the population. Therefore, a more complex game theoretic study, which involves the analysis of frequencydependent gene interactions (for example, neighbourmodulated fitness), is required.

Despite the potential key role of correlational selection among individuals in signal evolution the estimates of such effects are scant (Figure 1b). Correlational selection gradients are typically only estimated for traits within individuals (Kingsolver et al., 2001; Sinervo and Svensson, 2002), although progress is now being made on how female choice generates correlational selection on male traits (Blows et al., 2003; Figure 1c) or how male competition structures within individual correlation selection on signals and RHP traits (Figure 1a; McGlothlin et al., 2005). Only a single study has estimated the correlational selection between individuals. Sinervo and Clobert (2003) studied the impacts of the genetic similarity of neighbours (for example, multiple shared loci) on fitness of male colour morphs of Uta (Figure 1b). Our understanding of neighbour-modulated gene effects on multiple traits are sorely lacking. Theory often ignores the frequency dependence of neighbour-modulated effects (Wolf et al., 1999; Grafen, 2006). Furthermore, 
a
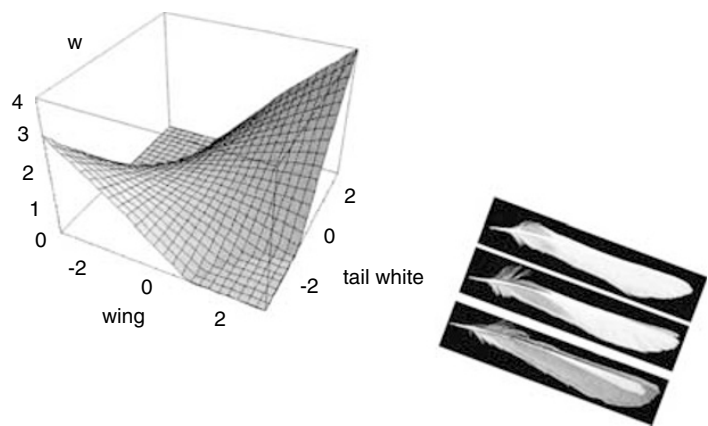

b
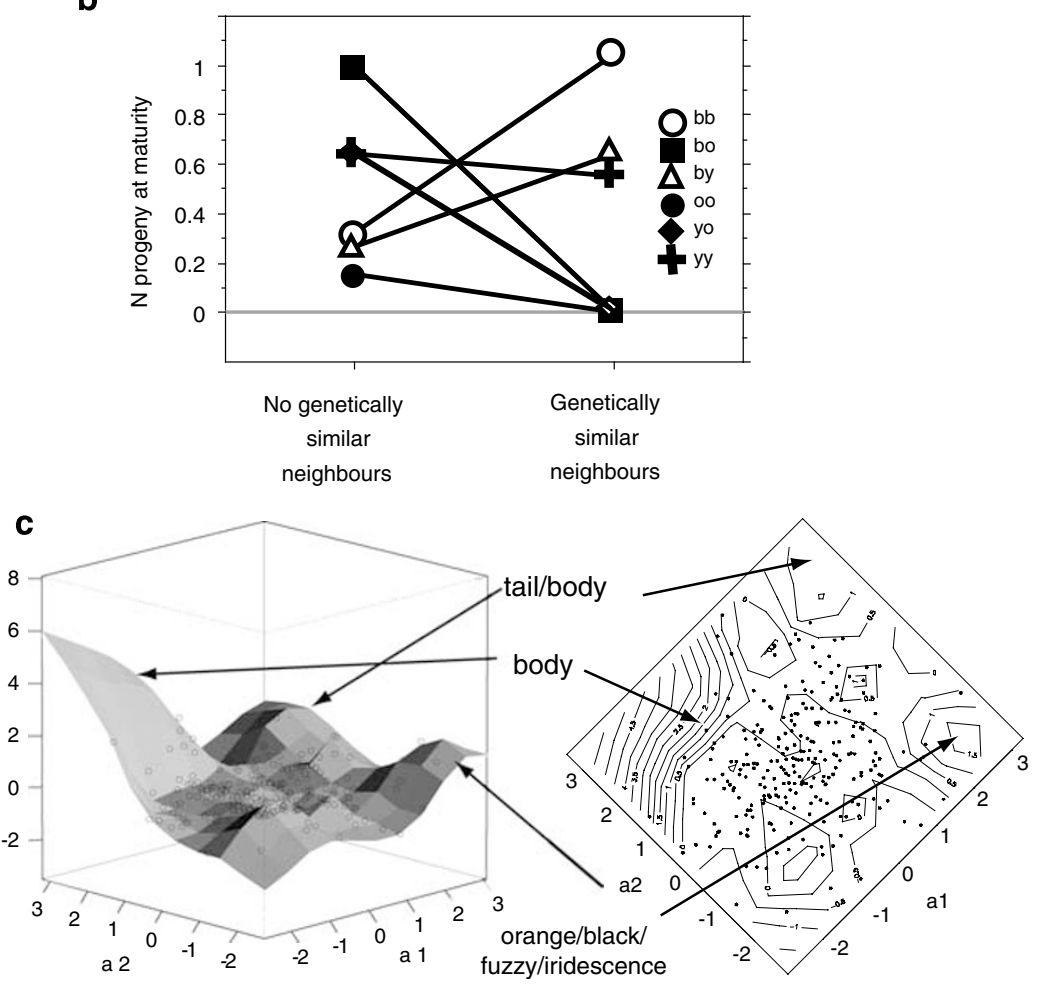

Figure 1 Examples of correlational selection (CS) (a) within vs (b) between individual traits/loci, or (c) within-individual traits driven by female choice. (a) Within individual CS on Resource Holding Potential (RHP) traits (size and tail white) in Junco hyemalis (McGlothlin et al., 2005). (b) Fitness consequences of the self-recognition and settlement behaviour in Uta stansburiana; an example of inter-individual CS. CS on recognition and settlement loci favours $\mathrm{B}=\mathrm{bb}$ males that settle next to genetically similar bb males. $\mathrm{O}$ males $(\mathrm{O}=\mathrm{oo}$, bo, yo) are favoured to settle next to genetically dissimilar males. Y (yy) is neutral for this behaviour (Sinervo and Clobert, 2008). (c) CS driven by female preference for male guppy, Poecilia reticulata, colour and morphology favours three distinct peaks of highly preferred males (from Blows et al., 2003).

current life history theory ignores the three combinations of correlation selection, enunciated above, and necessary coupling to frequency-dependent selection. The next phase of life history research should be directed at unravelling the social epistasis of correlational selection that is induced by social system trade-offs, and how social system trade-offs affect allocation trade-offs to competing functions. To study such effects correlational selection on traits must be estimated between individuals, as well as within individuals.

\section{The three levels of epistasis in social systems}

A unified view of epistasis recognizes a hierarchal level of interactions such that gene products build physiological systems, physiological systems interact to create signaller-receiver molecules of endocrine systems, endocrine systems control expression of multiple genes, signallers and receivers interact in social systems, and multiple genes (with variants at each locus) interact to create fitness surfaces and perhaps fitness epistasis (assessed by measuring correlational selection either within or between individuals). Physiological and behavioural epistases are unified; each involves senders and receivers. Physiological epistasis is intrinsic, whereas behavioural epistasis is extrinsic to organisms. Steroid hormones and DNA receptors such as hormone response elements (HREs) are a class of intrinsic physiological signals (Freedman and Luisi, 1993; Zajac and Chilco, 1995; Sanchez et al., 2002). However, hormones can also be used as extrinsic signals between individuals, such as in the case of maternal yolk steroids added to eggs of lizards (Lancaster et al., 2007) and birds (Schwabl, 1993) or steroid transfer between male and female progeny in 
the uterus of mammals (vom Saal et al., 1983). Such steroidal signals profoundly affect gene cascades in receivers.

Resolving any of the three levels of epistasis involves computation of a cross-product term (gene epistasis: Cordell, 2002, fitness epistasis: Whitlock et al., 1995, epistasis under frequency-dependent selection: see supplement to Sinervo and Calsbeek, 2006, or epistasis of physiological or hormonal effects: Lancaster et al., 2007). The cross-product term for traits under selection is called a correlational selection gradient (Phillips and Arnold, 1989).

\section{Genetic epistasis}

Genetic epistasis is related to physiological epistasis (Sinervo and Svensson, 2002; Sinervo and Calsbeek, 2003). In the case of gene epistasis within an individual, one gene interacts with another, perhaps shutting off that gene or alternatively, amplifying the products of a physiological pathway in a non-linear way (Cordell, 2002). In the case of signaller-receiver epistasis between individuals, alternative alleles at signal loci of a sender generate a receiver response that is dependent on alternative alleles at receiver loci and population frequency.

Epistasis among the genetic components of endocrine systems generates non-linear physiological effects and non-linear gene effects. Products of one gene may shut off other genes or promote the expression of a gene cascade. For example, sex-determining loci induce testis development and turn on genes for male traits (that is, the sry gene interacts with sox9; Koopman et al., 2001), which also downregulates other genes for female traits.

The additive effects of many individual pleiotropic genes are thought to generate the negative genetic correlations among life history traits. However, a continuum exists between levels of epistatic and additive variation depending on levels of additive, dominance and epistatic gene effects, and the relative levels of each component will also influence the magnitude of the genetic correlation due to additive vs epistatic causes. Levels of gene epistasis in nature are estimable with crosses, but to our knowledge it has only been estimated in crosses between populations or artificially selected lines (Bradshaw et al., 2005). The additive vs epistatic effects of genes can be partitioned with crosses within populations in the case of phenotype polymorphism due to two or more loci (Lenski, 1988; Lancaster et al., 2007).

\section{Physiological and behavioural epistasis}

Sewall Wright (1968) considered physiological epistasis (physiological or endocrine cascades) to be a universal property of genetic systems (Wade, 2002). He theorized that genetic variation in epistatic networks destabilized organismal function, and that epistatic variation is fixed in most species owing to negative effects of such complex gene networks on fitness. When epistatic genetic variation goes to fixation, such as when loci that contribute to epistases fix while others maintain segregating genetic variation, epistatic variation collapses creating additive genetic variation (Goodnight, 1995). As Wright hypothesized, epistatic gene effects may be fixed in many species that lack morphs.
However, sexual species have male and female morphs, and Wright's conjecture for epistasis is likely invalidated under strong sexual selection. This also has implications for more complex trade-offs that are expressed between the sexes, than are currently considered by life history theory. Males and females reflect the core morphs of sexual species. Recent advances in our understanding of life history trade-offs have identified different patterns of selection on the sexes as a source of additive genetic variation (Rice and Chippindale, 2001). Genetic trade-offs that promote functional trade-offs in organismal design between the sexes are referred to as inter-sexual ontogenetic conflict or intra-locus ontogenetic conflict (Rice and Chippindale, 2001). Alleles affecting male morphology and physiology and favoured by sexual selection are of limited value during natural selection on female morphology and physiology, and vice versa. Alleles should reach an optimum in each sex were it not for the fact that females and males repeatedly hybridize; they share genes in a common genome, except sex chromosomes (that is, the $\mathrm{Y}$ is restricted to males and the $\mathrm{X}$ resides in females $2 / 3$ of the time more often than in males; Gibson et al., 2002).

Ontogenetic conflict can evolve. Sequestering loci on sex chromosomes ameliorates the intensity of this tradeoff (Gibson et al., 2002). Alternatively, gene regulation by sex-limited steroid hormones, which govern expression of traits (and loci) in each sex, can evolve to ameliorate ontogenetic conflict (Sinervo and Calsbeek, 2003). Gene promoters (for example, HREs, see above) differentially control transcription and translation in the sexes under the control of sex steroids (Freedman and Luisi, 1993; Zajac and Chilco, 1995; Sanchez et al., 2002). Sex chromosomes, which initiate sex determination through gene cascades (for example, sry, sox9), are unlinked to autosomal genes where HRE reside. Thus, ontogenetic conflict, a life history trade-off between the sexes must arise from gene interactions or epistasis, not just pleiotropy.

Most life history analyses are restricted to one sex (that is, the female). The action of ontogenetic conflict is rarely studied, despite its importance to life history. Demonstrations of ontogenetic conflict are restricted to laboratory studies of fruit flies (Pischedda and Chippindale, 2006), or natural systems with pedigree on both sexes (red deer: Foerster et al., 2007; lizards: Calsbeek and Sinervo, 2004; Sinervo and McAdam, 2008). Ontogenetic conflict can be revealed as a negative genetic correlation between fitness of sires vs daughters (Foerster et al., 2007) and reciprocal lines of descent (dam-son, sire-daughter, Pischedda and Chippindale, 2006). Pedigree studies can reveal specific traits under ontogenetic conflict (for example, clutch size: Sinervo and McAdam, 2008, male size: Calsbeek and Sinervo, 2004 or dorsal patterns: Forsman and Appelqvist, 1995; Lancaster et al., 2007). However, to our knowledge, no study has yet resolved whether ontogenetic conflict arises from pleiotropic or epistatic effects of genes besides the genes for sex determination discussed above. Logically, the control of ontogenetic conflict arises from sex-determining loci and interactions with autosomal genes that modify the phenotype and thus, the fitness of each sex. However, gene mapping methods that are described below could be used to resolve the pleiotropic vs epistatic effects 
among genes that are activated by sex-determining loci (for example, for life history traits listed above: clutch size and body size).

Any species with alternative morphs (polymorphism) within a sex will potentially exhibit even greater levels of physiological epistasis and gene epistasis than a corresponding monomorphic congener. Morph loci, like sexdetermining loci, alter expression of endocrine pathways (Brantley et al., 1993). Morph loci consist of key regulatory genes of endocrine systems that organize suites of life history, behavioural, physiological and morphological traits into co-adapted syndromes. For example, loss-of-function genes create paedomorphic Axolotls (Ambystoma mexicanum) by mutations in the hypothalamic-pituitary-thyroid axis (Voss and Shaffer, 1997). In A. talpoideum, paedomorphs and metamorphs co-occur (Semlistch, 1998). Given the possibility of segregating epistatic variation within a population, morphs are of great interest to life history theory. Morphs are also under frequency-dependent selection, thereby invoking social system trade-offs. Systems with morphs may be key to elucidating the relative role of pleiotropy vs epistatis in life history trade-offs.

\section{Fitness epistasis: non-linear interactions of alleles} and effects on fitness

Genetic and physiological epistases are related to fitness epistasis (Whitlock et al., 1995; Kelly, 2000) in which nonlinear effects of traits on fitness are so extreme that alternative optima arise on fitness landscapes (Figure 1) In contrast, purely additive effects of traits (and alleles) generate much simpler (that is, one optimum) fitness landscapes (Sinervo and Svensson, 2002).

The examples of ontogenetic conflict (Figure 2) illustrate an interaction between genes on the sex chromosomes with other genes that code for the traits of each sex and that reside on the $X$ or autosomal chromosomes. Fitness epistasis can be observed in situations where obvious morphs are due to a simple genetic effect, and where other genes interact epistatically with such morph-determining loci. Often a morph locus may produce a signal that can be used by other morphs in the population to gain an advantage (for example, the colour loci of side-blotched lizards, the plumage loci of juncoes, the colour loci of guppies; Figure 1), thereby generating signaller-receiver epistasis. Within species, a specific morph locus that codes for morph determination may require interactions with other strategic loci to create ideal combinations of alleles (that is, functional integration, 'The genetic source(s) of life history tradeoffs') and high fitness for each morph (Sinervo and Clobert, 2003). RHP traits listed above (stamina, metabolism, immune function) illustrate some of the strategic loci that interact with the primary loci for morph determination.

The linkage between morph loci and social trade-offs is exemplified by the morphs of the side-blotched lizard, Uta stansburiana (Figure 1b). Orange males are aggressive within and between morphs, whereas blue males are cooperative within the blue type but aggressive towards the other two morphs (for example, towards both orange males or the cryptic sneaker males type with yellow throats). In this example, it is clear that genome-wide interactions of loci control cooperative blue and ultra- aggressive orange males of the lizard Uta. Blue males are favoured to share large numbers of alleles at many different loci (that is, high genetic similarity) with their social blue neighbours. In contrast, orange males are disfavoured to share any alleles with neighbours. The orange strategy, with high RHP, is favoured to be asymmetric in RHP with social neighbours. Conversely, cooperative strategies like that of blue males are favoured for symmetry in RHP, which stabilizes cooperation (Hamilton, 1964; Frank, 2003; Hochberg et al., 2003; Sinervo et al., 2006). Processes of correlational selection functionally integrate each social strategy in the Uta social system by sequestering alternative morph alleles of each morph with alleles at these alternative social strategies. Admixtures of these two morphs are constantly formed by sex (for example, segregation, recombination and gametic union), but fitness epistasis due to correlational selection results in the reformation of two fitness peaks for the number of alleles shared with a social neighbour (Figure 1b). The number of shared alleles in each morph alters patterns of survival and mating success of the different morphs thereby altering the life history trade-offs, but in a frequency-dependent fashion (Sinervo et al., 2006).

\section{Genetic methods for resolving trade-offs}

Social systems expand the number of traits and hence dimensions that are manifested as life history trade-offs. An outcome of interactions between males and females, or between other individuals, are a set of trade-offs among more traits than would be predicted by considering paired associations of traits, which is more typical of life history analysis. Behaviour per se imparts opportunities for multi-trait trade-offs to be generated through other processes besides ontogenetic conflict. For example, in the case of polygynandry, genetic effects of genomic imprinting or antagonistic sexual selection will alter life history trade-offs. Parental care, if present and obligate, will profoundly alter the kinds of life history trade-offs. Bi-parental care, if present but facultative in one sex, will generate strong frequencydependent selection. Other behavioural strategies like paternity assurance in males, fertility assurance or multiple paternity in females will likewise alter allocation to progeny (Calsbeek and Sinervo, 2004), and thus life history trade-offs. Sinervo and Clobert (2008) review these and other social system trade-offs and their impacts and demographic links to life history trade-offs.

Four methods have been used to assess pleiotropy or epistasis as a source of gene correlation that underlies life history trade-offs: (1) artificial selection on single life history traits and the correlated response on other life history traits, (2) genetic correlations among life history traits estimated with pedigree or genetic crosses, (3) gene engineering of a single life history trait and measuring the effect on other life history traits, (4) mapping multiple traits to one gene and verifying the action of pleiotropy, or refuting pleiotropy and verifying epistasis by mapping traits to interactions arising from multiple genes.

Below, we argue that only with results from gene mapping (method 4) can we discriminate between pleiotropy vs epistasis as the cause of life history trade- 

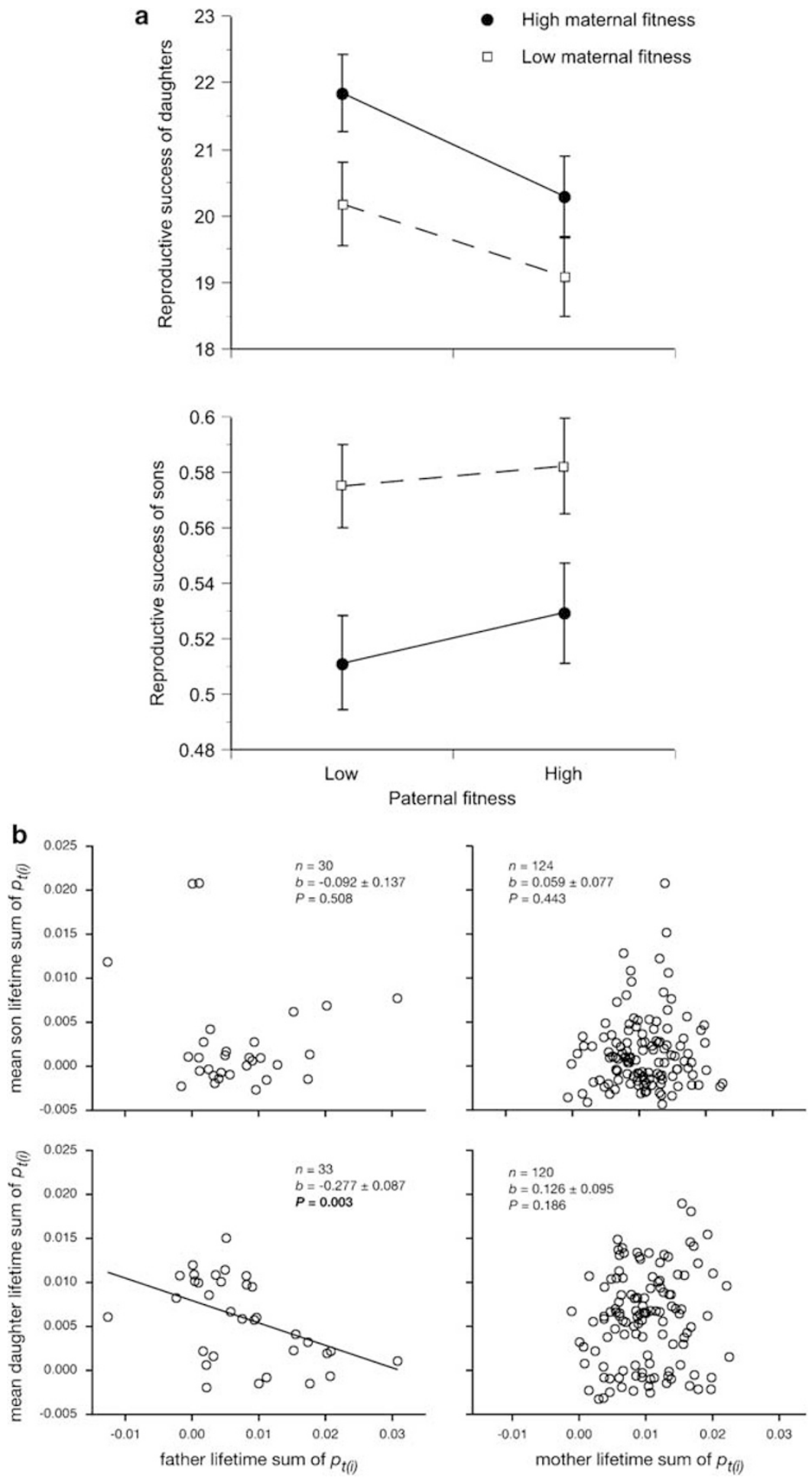

Figure 2 Ontogenetic conflict in Drosophila melanogaster (Pischedda and Chippindale, 2006) and red deer, Cervus elapus (Foerster et al., 2007) as measured by negative genetic correlations between the sexes.

offs. If artificial selection experiments (method 1) were devised to simultaneously select on two or more traits (and multiple loci), this method could in principle be used to estimate epistatic effects. Likewise, if gene engineering (method 2) was used to alter two independent loci that contribute to two or more life history traits, the epistatic source of life history trade-offs could also be elucidated. We also argue that estimating genetic correlations (method 3) is not very useful in resolving the distinction between pleiotropy vs epistasis as the cause of trade-offs, unless the method is augmented with explicit experiments. For example, experimental manipulations of a single endocrine system can be used to resolve the pleiotropic action of a single physiological system (Sinervo and Basolo, 1996). Experimental manipulations of two or more endocrine pathways (Lancaster 
et al., 2008; Mills et al., 2008) are required to resolve the epistatic action of multiple physiological (endocrine) systems, which govern the negative genetic correlation between life history traits.

\section{Artificial selection on one trait tugs on another, sexual selection tugs on many traits}

The action of pleiotropy can be resolved in artificial selection experiments as an associated genetic change in other traits that are not directly under selection (Lynch and Walsh, 1998). For example, artificial selection on early reproduction in Drosophila can concomitantly reduce lifespan in only a few generations (Rose and Charlesworth, 1981a,b). Although such approaches are informative in the lab, in nature genes are under multivariate selection.

Selection in the wild (Sinervo and Calsbeek, 2006) can create LD among unlinked genes (Lynch and Walsh, 1998). As noted above, a situation in which this often arises is during sexual selection that builds LD of sender (that is, male sexual signal) and receiver (that is, female choice) loci. Thus, artificial selection on male signals results in a correlated evolutionary response in female choice (Houde, 1994). The genetic correlation between female preference and male traits, which is demonstrated by artificial selection on guppies, must be due to unlinked loci as colour genes for male guppies reside on the Y chromosome (Houde, 1994). Sires pass on the colour genes that are located on the $\mathrm{Y}$ chromosome to sons. Genes that affect female preference, which females pass on to daughters, reside on an $\mathrm{X}$ chromosome or autosomes. Female preference (attraction to male) loci profoundly alter life history trade-offs through effects noted above for ontogenetic conflict.

Although artificial selection experiments are useful in uncovering pleiotropy, only in exceptional circumstances when the location of genes for given traits are known can artificial selection resolve epistases (for example, colour loci on Y chromosome of guppies; Houde, 1994). This is why gene mapping studies are crucial (method 4, below).

\section{Pleiotropy assessed by genetic correlations in a pedigree} and hormone manipulation

A second method to assess pleiotropy is to measure a negative genetic correlation between traits in a pedigree (Sinervo, 2000; Svensson et al., 2001) or in controlled crosses (Sinervo et al., 2001; Ernande et al., 2004). As noted in the Introduction, negative genetic correlations among phenotypic traits and components of fitness may arise from a pleiotropy.

Pedigree methods for elucidating ontogenetic conflict illustrate this principle (Figure 2). However, definitive proof of pleiotropy is not provided by a negative genetic correlation per se. As noted above, negative genetic correlations among life history traits can also arise from LD, which is reinforced by correlational selection (Sinervo and Svensson, 2002; Sinervo and Calsbeek, 2006), not simply pleiotropy. Experimental confirmation of pleiotropy can be obtained from manipulation of targeted endocrine pathways to perturb trade-offs (Sinervo and Basolo, 1996). Single manipulations cannot resolve epistasis.
More complex trade-offs of ontogenetic conflict are invoked by the endocrine regulation of life history traits in females and males when physiological systems are shared between the sexes (Figure 2; Sinervo and Calsbeek, 2003; Sinervo and McAdam, 2008). For example, hormones governing clutch size in females invoke life history trade-offs in females (for example, follicle-stimulating hormone and clutch size regulation induces costs of reproduction or the offspring sizeoffspring number trade-off; Sinervo and McAdam, 2008). However, genes for clutch size regulation invoke different pattern of trade-offs in male traits that are under sexual selection (Mills et al., 2008; Sinervo and McAdam, 2008). Genes for reproduction that are shared between the sexes (aside from a few sex-determining genes on Y or W chromosomes; Sinervo and Calsbeek, 2003) generate a key life history trade-off between the sexes, which is entirely ignored by the classic pleiotropic theory of life history trade-offs.

\section{Manipulating traits with gene manipulation and mapping traits to gene(s)}

A third method to identify pleiotropy is by gene manipulation (deletion or augmentation) or with transgenic lines (Leroi, 2001). Here we provide an example of gene manipulations that involve not only a life history trade-off (for example, energy allocated to reproduction vs non-reproductive function), but also a social system trade-off (for example, the costs and benefits of cooperation with non-self individuals).

Greenbeards are signaller-receiver loci that mediate social behaviours and life history trade-offs. A greenbeard is thought to consist of a pleiotropic social supergene (Hamilton, 1964; Dawkins, 1976) with pleiotropic effects on three traits: a signal, recognition of that signal, which triggers social acts like altruism to be directed to individuals expressing self signals (Sinervo et al., 2006), or agonistic acts to non-self individuals (Ross et al., 1996). Hamiltonian greenbeards pertain to concepts of kin relatedness, but apply them directly to single genes.

Consider the social amoeba, Dictyostelium discoideum, in which one gene controls greenbeard recognition (Queller et al., 2003). In social amoebae, high density induces individual amoeba to signal with cyclic AMP. On receiving this signal, amoebae aggregate at high concentration of cyclic AMP and build a fruiting body. Some cells commit to stalk that raise spores off the substrate. Other cells commit to spores. The $\operatorname{cs} A$ gene, a cell-surface adhesion protein, codes for a homophilic protein in cell membranes. Besides cyclic AMP, the CsA gene is key to aggregation behaviour. Deletion of $\operatorname{cs} A$ generates a mutant class of cheater cells that become fruiting body, at the expense of non-mutant cells (wild type), which get pushed into the stalk. This reflects an altruistic act that arises in the context of mutant cheaters. Only spores get fitness. The cheater advantage is however only realized in aggregations consisting of chimeras of mutant and wild-type cells. When allowed to self-aggregate on more natural substrates, homophilic (for example, self-loving) properties of $\operatorname{cs} A$ ensure that only the self-type (wild type) gains access to the fruiting body, thereby thwarting $\operatorname{cs} A$ mutants (for example, non-self). The $\operatorname{cs} A$ gene has pleiotropic effects on aggregation behaviour of cells into 
the multicellular slug, and $\operatorname{cs} A$ is involved in the selfrecognition behaviour that mediates social acts of altruism to self. Moreover, this social trade-off has direct impacts on competing life history allocations (reproductive structure vs reproductive tissue). Thus, links between selection at the level of altruistic social amoebae, multicellularity and life history trade-offs is clear; increased allocation to stalk to enhance propagule transmission impacts the number of amoebae that commit to an altruistic strategy of stalk vs spores.

\section{Gene mapping effects to a pleiotropic locus or an epistatic interaction of many loci}

A fourth and related genomic method is to use gene mapping technology to map suites of traits to one locus. The paedomorphosis example discussed above is a novel life history condition that affects suites of terrestrial adult traits. Adults mature in a larval form, which alters life history trade-offs (Semlitsch et al., 1988; Semlistch, 1998). The paedomorphic trait has been mapped to one locus in the Axolotl (Ambystoma mexicanum; Voss and Shaffer, 1997). To accomplish this gene mapping Voss and Shaffer (1997) crossed paedomorphic Axolotls to ancestral metamorphic A. tigrinum, and used linkage maps to follow associations of marker loci with expression of paedomorphosis in F1 and F2 progeny. Paedomorphosis, a recessive trait, is absent from the F1, but is reconstituted in F2 homozygotes, and its transmission is linked to one marker (Voss and Shaffer, 1997). Therefore, all of the life history trade-offs that are altered between paedomorphic vs metamorphic salamanders may be governed by the simple pleiotropic action of an endocrine gene. This does not however, preclude that additional loci might not be involved in epistatic tradeoffs. For example, in a population of polymorphic salamanders, frequency dependent and correlation selection on the paedomorphic vs metamorphic types may favour the build-up of alternative alleles for reproductive traits and the timing of reproduction that profoundly alters the life history trade-offs. The mapping of a single gene effect does not investigate any of the potential epistases.

Alternatively, pleiotropy is invalidated when traits involved in a trade-off are mapped to unlinked loci (Sinervo et al., 2006; Sinervo and Clobert, 2003). In this case, epistasis must be involved in trade-offs. The social system of male Uta (Figure $1 \mathrm{~b}$ ) resembles the dynamics of social amoebae in the way self-types self-assemble into cooperative groups, which thwarts the action of a class of cheaters. The blue lizards thwart yellow sneaker males. However, the blue cooperative strategy is vulnerable to invasion by the RHP strategy of orange males. Sinervo et al. (2006) mapped the colour signal locus for the greenbeard of the lizards and the self-recognition/aggregation loci (for example, of colour) to three other unlinked loci. Selection on loci for self-aggregation leads to non-linear (for example, epistatic) fitness effects (Figure 1b). In Uta, a distinct fitness peaks is present for both the orange and blue males (Figure 1b). Blue males are favoured for self-attraction to genetically similar neighbours whereas orange males are favoured for self-repulsion to genetically similar neighbours. Blue males aggregate next to genetically similar types. In contrast, orange males are hyperdispersed away from genetically similar types.

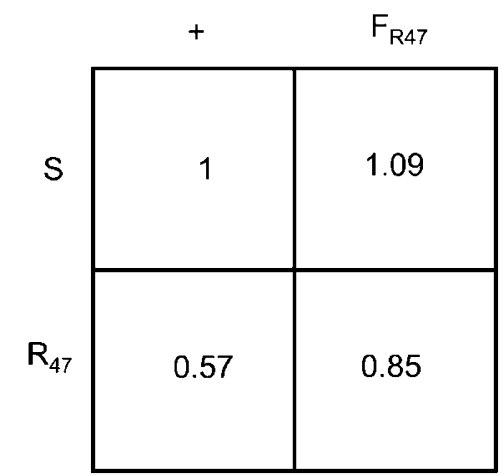

Figure 3 Relative fitness of four haploid genotypes of E. coli. $S$ and $R 47$ are alleles that confer sensitivity vs resistance to viruses $\mathrm{T} 4$ and T7. At another locus, + and $F_{R 47}$ are wild type and a strain that confers benefit to the $R 47$ genotype. Varying the fitness of $S / F_{R 47}$ radically alters the fitness topology; an example of fitness epistasis identified by constructing haploid genotypes of E. coli (from Lenski, 1988)

\section{Summary of the genetic methods for differentiating pleiotropy from epistasis}

In summary, traditional approaches of detecting life history trade-offs such as artificial selection, although effective in elucidating trade-offs among pairs of traits in the lab, cannot elucidate multi-variable trade-offs expressed in natural contexts, where LD can build due to sexual and social selection. It is entirely possible for artificial selection experiments to be designed to test for the epistatic source of life history trade-offs, but to our knowledge such experiments have not been carried out. Only in exceptional cases, where gene maps of traits exist (for example, guppy colour on the $\mathrm{Y}$ chromosome, female guppy preference on the $X$ chromosome or on autosomes), can artificial selection resolve gene epistasis. Although gene engineering is useful, its power in addressing pleiotropy vs epistasis in life history tradeoffs is limited. Gene engineering two loci can reveal fitness epistasis and circumvent this limitation. Lenski (1988), for example, manipulated two genes involved in resistance of E. coli to viral strains (Figure 3). Most gene engineering ignores gene epistasis, and the focus is often exclusively on pleiotropy. We suggest that more studies be devised to address the role of epistasis in life history trade-offs. Of the four methods, gene mapping a life history trade-off (or socially mediated life history tradeoff such as observed in amoeba or lizards) can directly uncover the epistatic vs pleiotropic source of life history trade-offs.

\section{Why pedigree methods will be critical in estimating trade-offs in nature}

Methods of transmission LD decay mapping (Lander and Green, 1987; Markianos et al., 2001), as we used in Uta (Sinervo et al., 2006), could be used to map the genetic architecture of life history trade-offs in nature (Slate et al., 2002). Even though the Uta linkage map is sparse (Box 1D), Sinervo et al. (2006) point out that loci associated with greenbeard behaviours (signal, recognition and donation) are distributed across the genome contrary to the standard view of greenbeards that favours an origin due to pleiotropy (Queller et al., 2003). Greenbeard aggregation behaviours of a social 


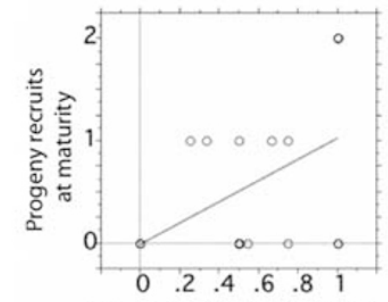

Frequency of yellow males in the social neighborhood

A. Mutualistic fitness benefit to $B$. Blue males with genetically similar neighbours benefit from a higher frequency of yellow in their local social neighbourhood, which increases the number of progeny $B$ males recruit to maturity.

Summary. This social situation is a pleiotropic consequence of the OBY signal being linked to testosterone regulation, and variation in stamina and RHP (Sinervo et al. 2000a), thereby invoking survival tradeoffs among males. O males have lower survival across years compared to $Y$ or $B$ males. (from Sinervo et al. 2006). To be effective in cooperation, $B$ males must share genes, especially genes for self-recognition and RHP traits (e.g., symmetry in RHP stabilizes cooperation). However, loci that generate genetic similarity (e.g., OBY locus, self-recognition, other RHP loci) are unlinked (see D), but interact epistatically. The mutualism of $B$ against $Y$ is traded-off against the altruism of $B$ against $O$, which is due to gene epistasis and pleiotropy.
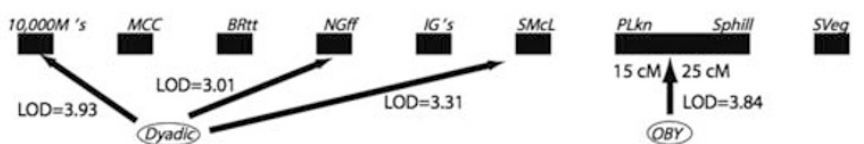

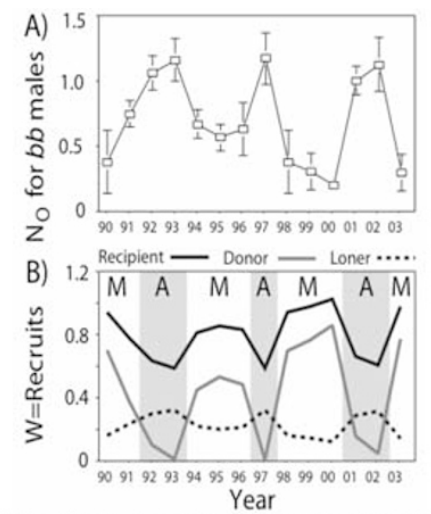

C. Cycle of $\mathrm{O}$ neighbours drives an evolutionary cycle of altruism and mutualism in B males. The black line shows the fitness of the recipient of altruistic actions in a male partnership of genetically similar B male neighbours. The grey line depicts fitness of altruist, relative to a loner B male strategy (dotted), which does not engage in group behaviours. When $O$ is common ( $\mathrm{A}$ for altruism) fitness of the B altruist (grey curve), which has more $O$ neighbours, dips below fitness of the loner B strategy (dotted curve). At these times B altruists should defect, and adopt a loner strategy. This egoistic behaviour has never been observed. When $O$ is rare ( $M$ for mutualism) $Y$ is common and both genetically similar B male neighbours obtain higher fitness than the loner B strategy and thus engage in evolutionary mutualism.

D. Gene mapping self-recognition loci. We mapped (Sinervo et al. 2006) loci for self-recognition, referred to as dyadic behavior. We scored behaviors in both sexes using a field pedigree. In males, we scored the propensity to settle beside genetically similar neighbors. In females, we scored the propensity to mate with genetically similar partners. We then determined which of the microsatellite markers was in physical linkage with genes responsible for self-recognition, using standard methods of gene mapping (Ott 1999). A primary linkage map must first be constructed for the microsatellite markers. Loci on separate blocks are unlinked $(e . g$., recombine, $r=1 / 2$ ) and are likely to reside on different chromosomes or on opposite arms of a large chromosome. The tendency of self-recognition behavior to be heritably transmitted to progeny is reflected in a marker locus that is transmitted in tight linkage. Loci with high LOD scores (Logarithm of Odds) sit on the same chromosome (e.g., physical linked) with the loci governing self-recognition (dyadic) behavior, or OBY colour. Three loci mapped close to self-recognition, of the 9 microsatellite loci. The OBY locus mapped between 2 loci (15 centiMorgans [cM] from 1 locus, $25 \mathrm{cM}$ from another). Therefore, genes that cause B male males to find and settle next to a genetically similar partner arise from the sharing of alleles at these 4 loci. We hypothesize that 1 locus must be related to a color preference for blue (Cc). We suspect that 1 locus is related to preference for males that have a self-similar pushup frequency $(P p)$ : B males spend a substantial fraction of the day directing push-ups to B partners. The 3 rd locus may be related to genes for the major histocompatibility complex (MHC) which is responsible for pheromone-based self-recognition (Ss) behaviour in vertebrates. The other loci (i.e., MCC, BRtt, IG, SVeg) that are not in physical linkage with self-recognition or OBY must sit next to genes that helps B males cooperate. We suspect these loci are related to down-regulation of aggression in cooperative B males (e.g., testosterone level, territoriality). However, alternative alleles that up-regulate aggression in $\mathrm{O}$ should also segregate at these strategic loci, named for their effect on male strategy. QTL map studies of territorial behaviour, body size, and plasma testosterone are underway,

supergene (Hamilton, 1964; Dawkins, 1976) need not merely be linked by pleiotropy, but greenbeard behaviours can self assemble from components of the genome, by the process of correlational selection acting on many self-attraction and self-aggregation loci (Figure 1b; Sinervo et al., 2006). These examples illustrate the unique genetic properties of behavioural traits, which can generate very interesting emergent properties that literally build a social system from simple gene interactions. This process is not just restricted to greenbeard traits, but our understanding of the role of behavioural genes is largely restricted to these case studies. The same process could in principle exist in any signaller-receiver context (with or without donation) for the case of any behaviour that is elicited in response to signal reception. For example, kin recognition and mate recognition could operate by the same types of loci (Sinervo et al., 2006). The key inference is to show that these signals and receiver behaviours invoke life history trade-offs, and to map the location of these genes to either pleiotropy or epistasis, with gene maps.

Elucidating the genomic architecture of life history trade-offs, which are modified and shaped by social trade-offs, requires pedigree methods. As noted above, lab studies cannot resolve the multi-dimensional nature of trade-offs invoked in social systems in nature. Gene maps can, but gene maps in field pedigrees are rare. Some have been linked to salient life history trade-offs. Slate et al. (2002) mapped quantitative trait loci (QTL) for progeny birth weight in red deer, which must be related to offspring quality and sexually selected trade-offs. Other pedigrees are available but have not yet been used to resolve life history traits. Beraldi et al. (2006) resolved several QTLs for traits in Soay sheep (coat colour and horns) using a primary linkage map based on 255 markers, but these gene maps have not been used to link obvious signals like coat colour or horns to the expression of life history trade-offs. Each of these pedigree studies is tantalizingly close to making additional inferences on the genomic architecture of life history trade-offs. We predict that epistasis will be rampant in nature and this epsistasis will profoundly alter life history trade-offs.

Current use of gene mapping technology is comparable to cartography in the Age of Discovery. We need to re-focus gene map technology on answering fundamental questions of genomic architecture of life history tradeoffs and gene regulation. In this way, gene mapping will 
move from simple cartography to understand the dimensions of genome structure, much like topography-revolutionized maps, or how the addition of the four dimensions of geologic layers by William Smith revolutionized our view of the earth's history (Winchester, 2001).

\section{Partitioning social system trade-offs with a price equation}

How do we estimate epistatic and socially mediated life history trade-offs in nature? Conceptual understanding of the causes of selection (Bock, 1977; Arnold, 1983) and life history trade-offs (Lande, 1983) has advanced through a multi-variate treatment of traits and fitness (Lande and Arnold, 1983; Frank, 1997). Phenotypic selection can be decomposed into directional and quadratic components. As noted above ('The three levels of epistasis in social systems'), significant cross-product terms test for the presence of epistasis (for example, gene, physiology or fitness). Correlational selection gradients are cross-product terms that describe interactions among phenotypic traits (Chevrud, 1984; Brodie, 1992; Sinervo and Svensson, 2002). Correlational selection gradients and the other more simple quadratic terms (for example, stabilizing and disruptive selection) describe the process of selection that generates functional integration between traits and loci (see 'The genetic source(s) of life history trade-offs', 'Social trade-offs, the life history and functional integration'), which govern life history trade-offs. Correlational selection gradients can also describe frequency-dependent selection between signals of senders, responses of receivers and their coevolutionary outcomes (Sinervo and Calsbeek, 2006 and its supplement). By co-evolution, we refer to the process by which the meaning of a signal evolves between two social actors.

For example, cooperative strategies will often evolve gene (signal) symmetry because RHP symmetry can stabilize cooperation (Frank, 2003; Sinervo et al., 2006). The meaning of the signal also evolves in the case of honest RHP signals used between social actors or in the case of a successful cheat strategy that evolves a deceptive signal to thwart RHP strategies. The correlational selection gradient estimated between individuals can be used to estimate this process of functional integration of the social system, analogous to the way in which correlational selection gradients within individuals capture functional integration of various competing physiological systems that invoke life history trade-offs (see 'The genetic source(s) of life history trade-offs').

In social systems, where social and life history tradeoffs become commingled due to signaller-receiver evolution (and the evolution of alternative signalling strategies), how can we resolve social trade-offs per se? The answer resides in group-structured versions of Price's equation (Gardner et al., 2007). The full PE is:

$$
\bar{w} \Delta \bar{z}=\operatorname{Cov}(w, z)]+E(w \Delta z)
$$

The PE is rarely used in its full form, which involves the term $E[w \Delta z]$ that describes changes in genome structure during transmission. The simple PE with the transmission term, $E(w \Delta z)$, deleted is standard in formulations of selection (for example, multi-variate selection, see Frank, 1997):

$$
\bar{w} \Delta \bar{z}=\operatorname{Cov}(w, z)
$$

where $w$ is fitness and $z$ is a phenotypic trait(s) under selection.

One of the problems with the full PE is that it lacks dynamic sufficiency (Grafen, 2006). The PE is a recursion equation. Transmission terms (on the right hand side of Equation (1): $E(w \Delta z)$ ) accept the $\Delta z$ of prior generations (for example, on the left hand side), producing an everexpanding series over evolutionary time. Given this dynamic insufficiency, there was no way to estimate the higher order moments that typify genetic variation under selection, such as the epistasis or gene correlations contained in the $E[w \Delta z]$ term. Quasi-linkage equilibrium (QLE) approximation as proposed by Gardner et al. (2007)(Barton and Turelli, 1987; Kirkpatrick et al., 2002) can achieve dynamic sufficiency. The PE can be used if selection creates weak LD, or if the alternative alleles are at low frequency during invasion. This advance allows solution of multi-locus games and thus epistatic trade-offs that impact life history traits of social species.

\section{Application of a group-structured version of the PE: a case study with Uta}

A partitioning of social trade-offs afforded by a groupstructured version of the PE has recently been applied to field pedigree. Sinervo et al. (2006) used a groupstructured version of the PE (equations therein). In Box 1 we provide a graphical depiction of the fitness gains and losses of the male morphs of Uta in neighbourhoods of genetically similar and dissimilar neighbours. These neighbourhoods form by a process of progeny dispersal and at maturity genes promote selfattraction to colour, which induce blue males to seek out and aggregate with males that share blue colour and selfattraction genes. Blue aggregations form by greenbeard recognition and attraction, not kin philopatry. Sinervo and Clobert (2003) used similarity of genes in neighbours (Sinervo and Clobert, 2003) as a proxy for self-similar genes that are required for cooperation to flourish (Figure 1b). We (Sinervo et al., 2006) mapped selfrecognition factors (that is, genes required to find genetically similar partners) to four specific loci (Box 1D), one of which was the colour locus. Finally, we computed fitness relations among groups of social actors as a function of genetic similarity and colour loci (Box 1).

Here, we focus on the social trade-offs of one of the morphs, the blue type, and for the moment ignore the social and life history trade-offs of orange and yellow. Blue males must cooperate to thwart the sneaky yellow male type, but this blue aggregation is vulnerable to the invasion of an orange type with high RHP, which extracts a fitness cost from one blue partner. Thus, survival and reproduction trade-offs of blue males involve interactions with three kinds of social neighbours (orange, blue and yellow). Orange on the other hand, has high mortality after reproduction and especially if orange is common. Thus, paternity success of orange invokes classic RHP trade-offs of low survival (Sinervo et al., 2000a). Yellow enjoys high survival if blue is rare because it forgoes resource allocation to territoriality, but 
yellow tends to sire progeny late in the season after other males die (Zamudio and Sinervo, 2000).

\section{Beyond group-structured PEs of selection and on to the transmission term}

The QLE approximation of weak selection is likely too stringent for intense selection observed in a social system like the Uta rock-paper-scissors (RPS) system. Numerical and game theoretic approaches are required (Alonzo and Sinervo, 2001, 2007). Even though QLE versions of Price's equation cannot capture the final phases of RPS dynamics with high levels of LD, it could be used to derive solutions of initial phases, when novel social systems evolve and LD is weak. The QLE versions of the PE could be used to partition genetic co-variation of traits related to life history trade-offs (sensu Lande, 1983) that are due to pleiotropy (additive) vs epistatic social causes. The partitioning of fitness for each male strategy of Uta illustrates the utility of the group-structured PE in analysing the social causes of life history trade-offs.

\section{Evolutionary games and the generality of epistatic social trade-offs}

The Uta social system can be viewed as a genic version of Axelrod and Hamilton's (1981) iterated Prisoner's Dilemma, but with the twist of another social actor besides the two prisoners and district attorney. The blue gene complex must be altruistic to weather cyclical phases of orange invasion. Orange plays the classic role of a district attorney in the IPD and enforces blue male altruism. By protecting blue recipients from orange, altruistic partners forgo fitness and the gene complex is passed down through a recipient's lineage, when in the next generation it is mutualistically beneficial against yellow. The blue recognition complex must be maintained intact to thwart cheats. Some yellow genotypes carry a single $b$ allele (that is, by genotype), but because they share few alleles at the other self-recognition loci required for cooperation, they are rejected from blue coalitions. The gene complex of blue recognition comprises secret handshakes of cooperation, which protect coalitions from cheats.

Amazingly, such rock-paper-scissors social systems are widespread. Crustaceans, insects, lizards, bacteria and humans exhibit variations on the RPS (Sinervo and Calsbeek, 2006; Sinervo et al., 2007; Figure 4). Many other species exhibit trimorphisms, which have not yet been verified to exhibit RPS dynamics (elephant seals, Gouldian finch, the ruff, trisytlous plants). Morphs are not necessary to generate epistatic trade-offs. Fisherian runaway and frequency-dependent selection is sufficient to generate ontogenetic conflict and epistatic trade-offs.

\section{Conclusions}

Until now, research in evolutionary biology has only focused on the selection term of the PE, but to understand the evolution of trade-offs, we need to assess how the transmission term of the PE equation changes over time. The transmission term is intimately related to the evolution of genetic correlations among traits, life history traits in particular. The PE transmission term can describe the evolution of pleiotropy and epistasis that generate social and life history trade-offs. We need to
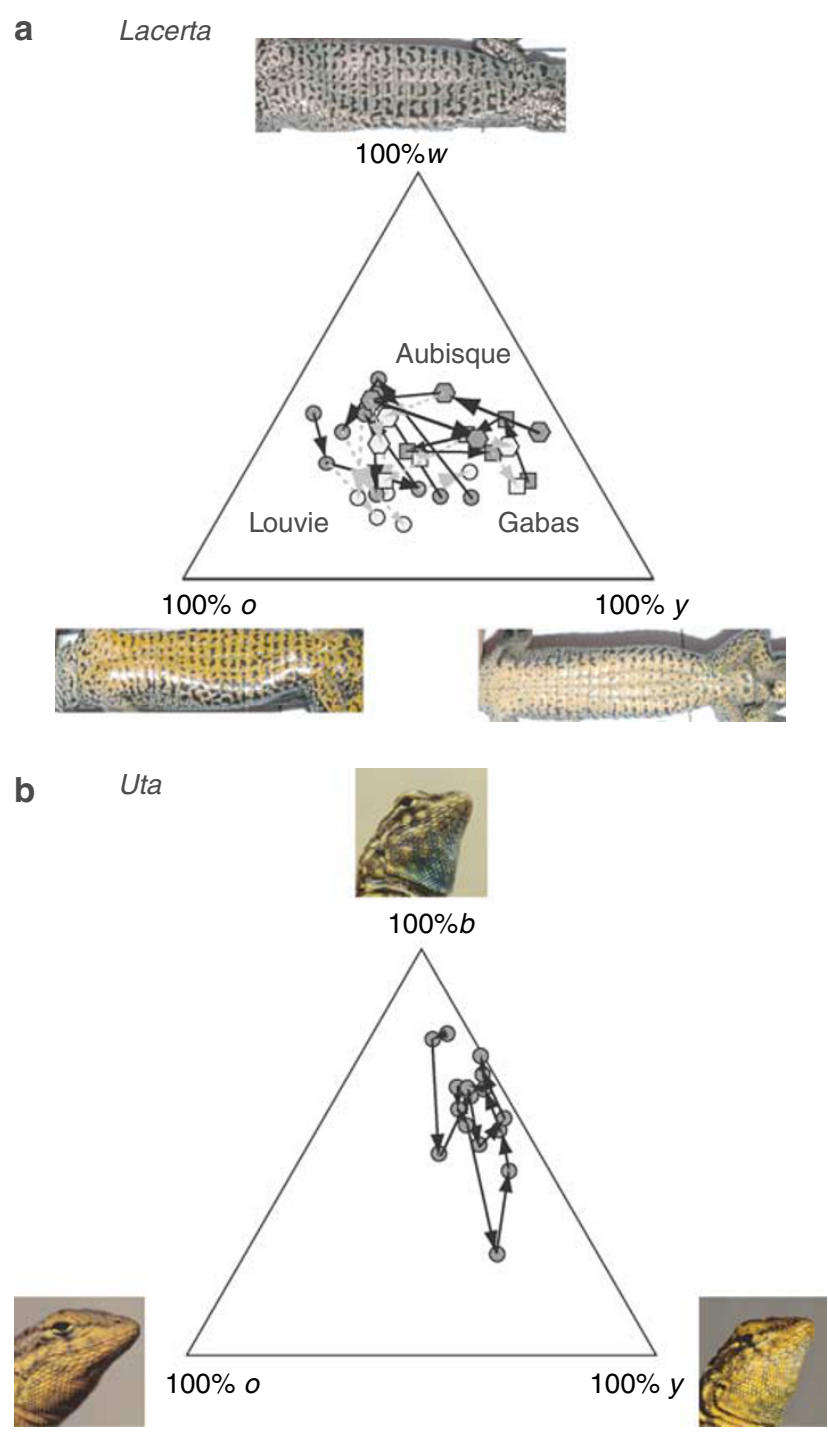

Figure 4 RPS cycles of colour alleles in Lacerta vivipara and Uta stansburiana appear to be driven by self-recognition loci (Sinervo et al., 2007). These two species have virtually the same colour signals and relationships among colour strategies despite last sharing a common ancestor 175 million years ago. RPS orbits for five populations are depicted for Lacerta (2002-2006: three at Louvie (circles), Aubisque (hexagons), Gabas (triangles)), and one population is shown for Uta (1990-2006). Both species exhibit rapid fourgeneration RPS orbits (from Sinervo et al., 2007).

study how epistases build from social selection (PE selection term), and how such epistases are converted to pleiotropy when social systems collapse and epistasis is converted to additive pleiotropy (that is, PE transmission term). One-strategy systems can be invaded and converted to a two-strategy system. Two-strategy systems can be invaded by a third. A three-strategy social system, can collapse back to two strategies, or collapse even further back to one strategy. When this occurs, new pleiotropic gene networks may result, and the new gene networks may form entirely new adaptive possibilities in further rounds of epistatic social selection. The pleiotropies (and epistases) in these new two and one strategy systems may differ fundamentally from the ancestral one and two strategy social systems. When this happens new 
trade-offs may evolve, freeing up evolutionary change from old constraints that previously kept the social system bounded.

How many dimensions are there for life history trade-offs? Social behaviours create complexities in trade-offs between pairs of traits within and between the sexes or among alternative types. How many life history trade-off dimensions are involved in social systems? This quantity has not yet been estimated for social systems. However, in the Uta social system we can enumerate at least six trade-off axes. Three trade-offs act on males: gene symmetry-asymmetry and cooperation vs conflict (Sinervo et al., 2006), survival vs polygamy (Sinervo and Lively, 1996; Sinervo et al., 2000a) and polygynyterritoriality (Zamudio and Sinervo, 2000). Two tradeoffs act on females: egg size-number (Sinervo et al., 1992), and costs of reproduction trade-offs (Lancaster et al., 2008), which both involve density-dependent competition (Sinervo et al., 2000b). At least one additional trade-off invokes the ontogenetic conflict of genes expressed in the sexes (Sinervo and McAdam, 2008). Although simpler social systems (that is, those with fewer strategies) may have reduced trade-off dimensions, social animals harbour trade-off dimensions greater than two if they are sexual, perhaps more if the species experiences density-dependent competition. We must abandon classic bi-dimensional views of trade-offs, which assume only pleiotropy, and scrutinize more dimensions in which trade-offs are structured by gene epistasis.

Social systems and stability of linkage disequilibrium The static view of pleiotropy should be replaced by a more dynamic view in which chronic LD and gene epistasis is responsible for trade-offs. In this case, social systems have far greater potential for evolution because trade-offs are due to multiple, potentially unlinked loci, which can fix for many more combinations of traits. Evolution need not proceed along pleiotropic trade-offs under this view, but more expansive evolutionary change is possible. Many different multi-locus combinations could fix in different species if traits are due to epistatic life history trade-offs. Under pleiotropy, fixation for two endpoints of a trade-off axis between two traits is the only possibility, which would greatly limit the scope of evolutionary change of a given social system. Although we have focussed on frequency-dependent strategies, identical arguments apply to density-dependent life history traits (Sinervo, 2000; Sinervo et al., 2007), which is well within the purview of classic life history theory. These principles apply to all social species. Life history trade-offs harbour a large, but ignored, epistatic component. The magnitude of these epistatic trade-offs will remain obscure unless we apply gene mapping methods to field pedigrees. If we discover that social epistasis is common in nature, we could construct laboratory experiments to identify the magnitude of socially driven life history trade-offs. Such endeavours will necessarily be multi-dimensional involving multitrait artificial selection, or artificial selection under frequency-dependent selection.

\section{Acknowledgements}

This paper was prepared in part during a visiting Professorship awarded by Toulouse University to BS. We also thank Etienne Danchin, Andy Gardner and Alexis Chaine for stimulating discussions on this topic, and especially Alexis Chaine and two anonymous reviewers for constructive comments on previous drafts of this paper. Research was supported by an NSF grant awarded to BS and AGM.

\section{References}

Ahtiainen JJ, Alatalo RV, Kortet R, Rantala MJ (2005). A tradeoff between sexual signaling and immune function in a natural population of the drumming wolf spider Hygrolycosa rubrofasciata. J Evol Biol 18: 985-991.

Alonzo S, Sinervo B (2001). Mate choice games, contextdependent good genes, and genetic cycles in the sideblotched lizard Uta stansburiana. Behav Ecol Sociobiol 49: 176-186.

Alonzo S, Sinervo B (2007). The effect of sexually antagonistic selection on adaptive sex ratio allocation. Evol Ecol Res 9: 1-21.

Arnold SJ (1983). Morphology, performance and fitness. Am Zool 23: 347-361.

Axelrod R, Hamilton WD (1981). The evolution of cooperation. Science 211: 1390-1396.

Barton NH, Turelli M (1987). Natural and sexual selection on many loci. Genetics 127: 229-255.

Beraldi D, McRae AF, Gratten J, Slate J, Visscher PM, Pemberton JM (2006). Development of a linkage map and mapping of phenotypic polymorphisms in a free-living population of Soay sheep (Ovis aries). Genetics 173: 1521-1537.

Blows MW, Brooks R, Kraft PG (2003). Exploring complex fitness surfaces: mutliple orientation and polymorphism in male guppies. Evolution 57: 1622-1630.

Bock WJ (1977). Toward an ecological morphology. Vogelwarte 29: 127-135.

Bradshaw WE, Haggerty BP, Holzapfel CM (2005). Epistasis underlying a fitness trait within a natural population of the pitcher-plant mosquito, Wyeomyia smithii. Genetics 169: 485-488.

Brantley RK, Wingfield JC, Bass AH (1993). Sex steroid levels in Porichthys notatus, a fish with alternative reproductive tactics, and a review of the hormonal bases for male dimorphism among teleost fishes. Horm Behav 27: 332-347.

Brodie EDI (1992). Correlational selection for color pattern and antipredator behavior in the garter snake Thamnophis ordinoides. Evolution 46: 1284-1298.

Calsbeek R, Sinervo B (2004). Within clutch variation in offspring sex determined by differences in sire body size: cryptic mate choice in the wild. J Evol Biol 17: 464-470.

Charmantier A, Perrins C, McCleery RH, Sheldon BC (2006). Quantitative genetics of age at reproduction in wild swans: Support for antagonistic pleiotropy models of senescence. Proc Natl Acad Sci USA 103: 6587-6592.

Chevrud JM (1984). Quantitative genetics and developmental constraints on evolution by selection. J Theor Biol 110: 155-171.

Cordell HJ (2002). Epistasis-what it means, what it doesn't mean, and statistical methods to detect it in humans. Hum Mol Genet 11: 2463-2469.

Dawkins R (1976). The Selfish Gene. Oxford University Press: New York and Oxford.

Ernande B, Boudry P, Clobert J, Haure J (2004). Plasticity in resource allocation based life history traits in the Pacific oyster, Crassostrea gigas. I. Spatial variation in food abundance. J Evol Biol 17: 342-356.

Ferrière R, Clobert J (1992). Evolutionary stable age at first reproduction in a density-dependent model. J Theor Biol 157: 253-267. 
Foerster K, Coulson T, Sheldon B, Pemberton JM, Clutton-Brock TH, Kruuk LEB (2007). Sexually antagonistic genetic variation in the red deer. Nature 447: 1107-1111.

Forsman A, Appelqvist S (1995). Experimental manipulation reveals differential effects of colour pattern on survival in male and female grasshoppers. J Evol Biol 12: 391-401.

Frank SA (1997). The Price equation, Fisher's fundamental theorem, kin selection, and causal analysis. Evolution 51: 1712-1729.

Frank SA (2003). The repression of competition and the evolution of cooperation. Evolution 57: 693-705.

Freedman LP, Luisi BF (1993). On the mechanism of DNAbinding by nuclear hormone receptors: a structural and functional perspective. J Cell Biochem 51: 140-150.

Gardner A, West SA, Barton NH (2007). The relation between multilocus population genetics and social evolution theory. Am Nat 169: 207-226.

Gibson JR, Chippindale AK, Rice WR (2002). The X chromosome is a hot spot for sexually antagonistic fitness variation. Proc R Soc Lond Ser B Biol Sci 269: 499-505.

Goodnight CJ (1995). Epistasis and the increase in additive genetic variance: Implications for phase 1 of Wright's shifting-balance process. Evolution 49: 502-511.

Grafen A (2006). Optimization of inclusive fitness. I Theor Biol 238: 541-563.

Hamilton WD (1964). The evolution of social behavior. J Theor Biol 7: 1-52.

Hamilton WD, May RM (1977). Dispersal in stable habitats. Nature 269: 578-581.

Hochberg ME, Sinervo B, Brown SP (2003). Socially mediated speciation. Evolution 57: 154-158.

Houde AE (1994). Effect of artificial selection on male colour patterns and mating preference of female guppies. Proc $R$ Soc Lond B Biol Sci 256: 125-130.

Kelly JK (2000). Epistasis, linkage and balancing selection. In: Wolf JB, Brodie III ED, Wade MJ (eds). Epistasis and Evolutionary Process. Oxford University Press: New York, NY. pp 146-157.

Kingsolver JG, Hoekstra HE, Hoekstra JM, Berrigan D, Vignieri SN, Hill CE et al. (2001). The strength of phenotypic selection in natural populations. Am Nat 157: 245-261.

Kirkpatrick M, Johnson T, Barton NH (2002). General models of multilocus evolution. Genetics 161: 1727-1750.

Kokko H (2001). Fisherian and 'good genes' benefits of mate choice: how (not) to distinguish between them. Ecol Lett 4: 322-326.

Kokko H, Jennions MD, Brooks R (2006). Unifying and testing models of sexual selection. Ann Rev Ecol Evol Syst 37: 43-66.

Komdeur J, Hatchwell BJ (1999). Kin recognition: function and mechanism in avian societies. Trends Ecol Evol 14: 237-241.

Koopman P, Bullejos M, Bowles J (2001). Regulation of male sexual development by Sry and Sox9. J Exp Zool 290: 463-474.

Lancaster L, Hazard LC, Clobert J, Sinervo BR (2008). Corticosterone manipulation reveals differences in hierarchical organization of multidimensional trade-offs in r-strategists and K-strategist females. J Evol Biol 21: 556-565.

Lancaster LT, McAdam AG, Wingfield JC, Sinervo B (2007). Adaptive social and maternal induction of antipredator dorsal patterns in a lizard with alternative social strategies. Ecol Lett 10: 798-808.

Lande R (1983). A quantitative genetic theory of life history evolution. Ecology 63: 607-615.

Lande R, Arnold SJ (1983). The measurement of selection on correlated characters. Evolution 37: 1210-1226.

Lander ES, Green P (1987). Construction of multilocus geneticlinkage maps in humans. Proc Natl Acad Sci USA 84: 23632367.

Lenski RE (1988). Experimental studies of pleiotropy and epistasis in Escherichia coli. Compensation for maladaptive effects associated with resistance to virus T4. Evolution 42: 433-440.

Leroi AM (2001). Molecular signals versus the Loi de Balancement. Trends Ecol Evol 16: 24-29.

Lynch M, Walsh B (1998). Genetics and Analysis of Quantitative Traits. Sinauer Associates, Inc.: Sunderland, MA, USA.

Markianos K, Daly MJ, Kruglyak L (2001). Efficient multipoint linkage analysis through reduction of inheritance space. Am J Hum Genet 68: 963-977.

Maynard Smith J (1982). Evolution and the Theory of Games. Cambridge University Press: Cambridge.

McGlothlin JW, Parker PG, Nolan V, Ketterson ED (2005). Correlational selection leads to genetic integration of body size and an attractive plumage trait in dark-eyed juncos. Evolution 59: 658-671.

Miles DB, Sinervo B, Hazard L, Svensson EI, Costa D (2007). Relating endocrinology, physiology and behaviour using species with alternative mating strategies. Funct Ecol 21: 653-665.

Miller BL, Sinervo B (2007). Heritable body size mediates apparent life-history trade-offs in a simultaneous hermaphrodite. J Evol Biol 20: 1554-1562.

Mills SM, Hazard L, Lancaster L, Mappes J, Miles DB, Oksanen TA et al. (2008). Gonadotropin hormone modulation of testosterone, immune function, performance and behavioral trade-offs among male morphs of the lizard, Uta stansburiana. Am Nat 171: 339-357.

Phillips PC, Arnold SJ (1989). Visualizing multivariate selection. Evolution 43: 1209-1222.

Pischedda A, Chippindale AK (2006). Intralocus conflict diminishes the benefits of sexual selection. PloS Biol 4: e356.

Queller DC, Ponte E, Bozzaro S, Strassmann JE (2003). Singlegene greenbeard effects in the social amoeba Dictyostelium discoideum. Science 299: 105-106.

Reznick D, Nunny L, Tessier A (2000). Big houses, big cars, superfleas, and the cost of reproduction. Trends Ecol Evol 15: 421-425.

Rice WR, Chippindale AK (2001). Intersexual ontogenetic conflict. J Evol Biol 14: 685-693.

Roff DA, Fairbairn DJ (2006). The evolution of trade-offs: where are we? J Evol Biol 20: 433-447.

Rose M, Charlesworth B (1981a). Genetics of life history in Drosophila melanogaster. I. Sib analysis of adult females. Genetics 97: 173-186.

Rose M, Charlesworth B (1981b). Genetics of life history in Drosophila melanogaster. II. Exploratory selection experiments. Genetics 97: 187-196.

Ross KG, Vargo EL, Keller L (1996). Simple genetic basis for important social traits in the fire ant Solenopsis invicta. Evolution 50: 2387-2399.

Sanchez R, Nguyen D, Rocha W (2002). Diversity in mechanisms of gene regulation by estrogen receptors. Bioessays 24 : 244-254.

Schwabl H (1993). Yolk is a source of maternal testosterone for developing birds. Proc Natl Acad Sci USA 90: 11446-11450.

Semlistch RD (1998). Intraspecific heterochrony and life history: decoupling somatic and sexual development in a facultatively paedomorphic salamander. Proc Natl Acad Sci USA 95: 5643-5648.

Semlitsch RD, Scott DE, Pechmann JHK (1988). Time and size at metamorphosis related to adult fitness in Ambystoma talpoideum. Ecology 69: 184-192.

Shuster SM, Wade MJ (2003). Mating Systems and Strategies. Princeton University Press: Princeton, NJ, USA.

Sinervo B (1999). Mechanistic analysis of natural selection and a refinement of Lack's and William's principles. Am Nat 154: S26-S42.

Sinervo B (2000). Adaptation, natural selection, and optimal life history allocation in the face of genetically-based trade-offs. In: Mousseau T, Sinervo B, Endler JA (eds). Adaptive Genetic Variation in the Wild. Oxford University Press: Oxford, UK, pp 41-64. 
Sinervo B, Basolo AL (1996). Testing adaptation using phenotypic manipulations. In: Rose MR, Lauder GV (eds). Adaptation. Academic Press: New York. pp 149-185.

Sinervo B, Bleay C, Adamopoulou C (2001). Social causes of correlational selection and the resolution of a heritable throat colour polymorphism in a lizard. Evolution 240: 2040-2052.

Sinervo B, Calsbeek R (2003). Physiological epistasis, ontogenetic conflict and natural selection on physiology and life history. Integr Comp Biol 43: 419-430.

Sinervo B, Calsbeek R (2006). The developmental, physiological, neural and genetical causes and consequences of frequency dependent selection in the wild. Ann Rev Ecol Evol Syst 37: 581-610.

Sinervo B, Chaine A, Clobert J, Calsbeek R, Hazard L, Lacncaster L et al. (2006). Colour morphs and genetic cycles of greenbeard mutualism and transient altruism. Proc Natl Acad Sci USA 102: 7372-7377.

Sinervo B, Clobert J (2003). Morphs, dispersal, genetic similarity and the evolution of cooperation. Science 300: 1949-1951.

Sinervo B, Clobert J (2008). Life history strategies, multidimentional trade-offs and behavioural syndromes. In: Danchin E, Giraldeau L-A, Cézilly F (eds). Behavioural Ecology. Oxford University Press: Oxford, pp 135-183.

Sinervo B, Doughty P, Huey RB, Zamudio K (1992). Allometric engineering: a causal analysis of natural selection on offspring size. Science 258: 1927-1930.

Sinervo B, Heulin B, Surget-Groba Y, Clobert J, Miles DB, Corl A et al. (2007). Models of density-dependent genic selection and a new rock-paper-scissors social system. Am Nat 170: 663-680.

Sinervo B, Lively CM (1996). The rock-paper-scissors game and the evolution of alternative male reproductive strategies. Nature 380: 240-243.

Sinervo B, McAdam AG (2008). Maturational costs of reproduction and ontogenetic conflict between the sexes: genetic and experimental assessment of cryptic natural selection on clutch size in the wild. Proc $R$ Soc Lond B Biol Sci 275: 629-638.

Sinervo B, Miles DB, DeNardo D, Frankino T, Klukowski M (2000a). Testosterone, endurance and Darwinian fitness: natural and sexual selection on the physiological bases of alternative male behaviours in side-blotched lizards. Horm Behav 38: 222-233.

Sinervo B, Svensson E (2002). Correlational selection and the evolution of genomic architecture. Heredity 89: 329-338.
Sinervo B, Svensson E, Comendant T (2000b). Density cycles and an offspring quantity and quality game driven by natural selection. Nature 406: 985-988.

Slate J, Visscher PM, MacGregor S, Stevens D, Tate ML, Pemberton JM (2002). A genome scan for quantitative trait loci in a wild population of red deer (Cervus elaphus). Genetics 162: 1863-1873.

Svensson E, Sinervo B, Comendant T (2001). Density-dependent competition and selection on immune function in genetic lizard morphs. Proc Natl Acad Sci USA 98: 12561-12565.

Svensson EI, Sinervo B, Comendant T (2002). Mechanistic and experimental analysis of condition and reproduction in a polymorphic lizard. I Evol Biol 15: 1034-1047.

Thompson JN (2005). The Geographic Mosaic of Coevolution. University of Chicago Press: Chicago.

vom Saal FS, Grant WM, McMullen CW, Laves KS (1983). High fetal estrogen concentrations: correlations with increased sexual activity and decreased aggression in male mice. Science 220: 1306-1309.

Voss SR, Shaffer HB (1997). Adaptive evolution via a major gene effect: paedomorphosis in the Mexican axolotl. Proc Natl Acad Sci USA 94: 14185-14189.

Wade MJ (2002). A gene's eye view of epistasis, fitness and speciation. J Evol Biol 15: 337-346.

Wagner G, Altenberg L (1996). Complex adaptations and the evolution of evolvability. Evolution 50: 967-976.

Whitlock MC, Phillips PC, Moore FBG, Tonsor SJ (1995). Multiple fitness peaks and epistasis. Ann Rev Ecol Syst 26: 601-629.

Winchester S (2001). The Map that Changed the World: William Smith and the Birth of Modern Geology. Harper Collins: New York, NY, USA.

Wolf JB, Brodie III ED, Moore AJ (1999). Interacting phenotypes and the evolutionary process. II. Selection resulting from social interactions. Am Nat 153: 254-266.

Wright W (1968). Evolution and the Genetics of Populations. Chicago University Press: Chicago, IL, USA.

Zajac JD, Chilco PJ (1995). Transcriptional control and the regulation of endocrine genes. Clin Exp Pharmacol Physiol 22: 935-943.

Zamudio K, Sinervo B (2000). Polygyny, mate-guarding, and posthumous fertilizations as alternative male strategies. Proc Natl Acad Sci USA 97: 14427-14432.

Zera AJ, Harshman LG (2000). The physiology of life history trade-offs in animals. Ann Rev Ecol Syst 32: 95-126. 